\title{
Localized Marked Elongation of the Distal Internal Carotid Artery with or without PHACE Syndrome: Segmental Dolichoectasia of the Distal Internal Carotid Artery
}

\author{
(iD)Z.Y. Jia, DL.B. Zhao, and (DD.H. Lee
}

\begin{abstract}
BACKGROUND AND PURPOSE: Segmental intracranial dolichoectasia of the distal ICA is a feature of PHACE syndrome or a sporadic phenomenon. We evaluated the relationship between intracranial dolichoectasia of the distal ICA and PHACE syndrome and illustrated the characteristic radiologic findings of the lesions.
\end{abstract}

MATERIALS AND METHODS: Intracranial dolichoectasia of the distal ICA was identified in 20 patients at our institution from 2005 to 2016 through a review of diagnostic cerebral angiography results. All radiologic images were reviewed to determine the vascular morphologic dispositions around the distal ICA, including dysplasia, mural calcification, vessel wall enhancement, lumen narrowing, and aneurysm formation. Medical records were reviewed to determine the symptoms of PHACE syndrome. Subsequently, the correlation between radiologic findings and PHACE syndrome was assessed.

RESULTS: In this cohort, which had a strong female predominance (male/female ratio= 2:18), intracranial dolichoectasia had a more ipsilateral vascular morphologic disposition. Mural calcification was detected more frequently in elderly patients, whereas vessel wall enhancement was detected more frequently in younger patients. Follow-up images showed a slow progression of the lesions. However, no significant differences in the vascular morphologic disposition and brain structural changes were observed between patients with $(n=$ $11)$ and without $(n=9)$ PHACE syndrome.

CONCLUSIONS: The striking elongation and tortuosity of the distal ICA generally appeared to be a type of congenital lesion occurring early in embryogenesis as either a sporadic phenomenon or an arterial change associated with PHACE syndrome. Imaging findings revealed various mural abnormalities with a benign clinical course.

ABBREVIATIONS: AchoA = anterior choroidal artery; $\mathrm{BA}=$ basilar artery; $\mathrm{CS}=$ communicating segment; ICDE = intracranial dolichoectasia; $\mathrm{PCA}=$ posterior cerebral artery; PcomA = posterior communicating artery; PHACE = posterior fossa malformations, hemangiomas, arterial anomolies, cardiac defects, and eye abnormalities; $\mathrm{OA}=$ ophthalmic artery

M arked arterial elongation and tortuosity (intracranial dolichoectasia [ICDE]) have been reported previously as common features of PHACE syndrome $e^{1-4}$ or as a sporadic phenomenon. ${ }^{5-7}$ PHACE syndrome refers to conditions associated with posterior fossa malformations, hemangiomas, arterial anomolies, cardiac defects, and eye abnormalities. ${ }^{8}$ ICDE of the intracranial arteries is one of the various features of the arterial cerebrovascular abnormalities.

Received July 29, 2017; accepted after revision January 1, 2018

From the Department of Radiology and Research Institute of Radiology (Z.Y.J., L.B.Z., D.H.L.), Asan Medical Center, University of Ulsan College of Medicine, Seoul, Korea; and Department of Radiology (Z.Y.J., L.B.Z.), The First Affiliated Hospital of Nanjing Medical University, Jiangsu Province, China.

Please address correspondence to Deok Hee Lee, MD, PhD, Department of Radiology and Research Institute of Radiology, Asan Medical Center, University of Ulsan College of Medicine, 88, Olympic-ro 43-gil, Songpa-gu, Seoul 138-736, Korea; e-mail: dhlee@amc.seoul.kr

三 Indicates article with supplemental on-line table.

http://dx.doi.org/10.3174/ajnr.A5573
On cerebrovascular imaging, from time to time, we encounter striking arterial elongations, particularly the distal ICA elongation, regardless of the PHACE syndrome status. This pathologic arterial elongation often co-occurs with marked tortuosity and may even appear as a conglomerated vascular mass to compensate for the limited length of the allocated arterial segment. It is often accompanied by conditions such as multiple stenoses, dilations, or aneurysms and usually features a variable amount of mural calcification. ${ }^{2}$ Angiographically, this vascular morphologic disposition exhibits a more congenital appearance because the unique features cannot be explained by an acquired anatomic alteration. Although these types of arterial changes may present in any arterial segment of the body, we focused on cases of segmental involvement of the distal ICA because in our angiographic experience (limited to the cerebrovascular system), this site is the most frequently affected.

The easily recognizable symptoms and clinical significance of the above-mentioned vascular morphologic disposition completely dif- 
fer from those of acquired nonsegmental dilative arteriopathy, which is frequently observed in the basilar artery (BA). Accordingly, this study describes angiographic and other imaging characteristics of this peculiar anatomic disposition, particularly in the distal ICA, by reviewing the radiologic findings and medical records of affected patients. We aimed to evaluate the possible associations of the radiologic findings with PHACE syndrome and illustrate the clinical follow-up results of the imaging morphologic abnormalities.

\section{MATERIALS AND METHODS}

\section{Definition of ICDE and Segmentation of the ICA}

Because pathologic arterial tortuosity cannot be quantified, we subjectively defined segmental ICDE by comparing other arterial segments, particularly those in the same segment on the contralateral side, as unusually marked areas of dolichosis with variable degrees of ectasia on the affected side. Notably, these areas could be easily demarcated from other adjacent segments with normal lengths and tortuosities.

We applied the 7-segment system proposed by Lasjaunias et $\mathrm{al}^{9}$ for the ICA to our analysis of the involved segments of the ICA and intracranial arteries. This embryology-based 7-segment system terminates at the origin of the posterior communicating artery (PcomA). Because the system does not name the segment of the adult ICA between the PcomA origin and ICA bifurcation into the MCA and anterior cerebral artery, we designated this specific segment as the "communicating segment" (CS) (Fig 1).

\section{Patient Population and PHACE Syndrome Diagnosis}

At our institution (Asan Medical Center), 11,516 patients underwent diagnostic cerebral angiography between January 2005 and December 2016. For our study, we limited the number of candidate cases by applying the search terms "carotid," "ICA," "dysplasia," and "dolichoectasia" and further limited the number to 45 patients by reviewing the angiography reports. Furthermore, all angiographic images of the included cases were reviewed to identify intracranial ICA abnormalities, including marked elongation and tortuosity. Finally, 20 patients were identified.

This retrospective review was approved by our institutional review board, and the requirement for individual patient consent was waived. The included patients' medical records were reviewed for symptoms and indications of PHACE syndrome and other significant disorders. All data obtained via radiologic imaging modalities (CT, CTA, MR imaging, TOF-MRA, contrast-enhanced MR vessel imaging, and brain perfusion SPECT) were reviewed in our PACS system by 2 radiologists (Z.Y.J. and L.B.Z.). If the individual radiologic analyses differed, the reviewers reached a consensus after discussion with a third reviewer (D.H.L.). Finally, the patients were stratified according to the "Consensus Statement on Diagnostic Criteria for PHACE Syndrome” of 2009 as follows: 1) PHACE syndrome, 2) possible PHACE syndrome, or 3 ) none. ${ }^{8}$

\section{Image Findings and Statistical Analysis}

After recording the laterality and segments of the involved ICA, the morphology of the arterial components around the distal ICA was recorded by reviewing DSA, CTA, and MRA images of the anterior cerebral artery (A1 segment), anterior communicating artery, MCA (M1), anterior choroidal artery (AchoA), PcomA, ophthalmic

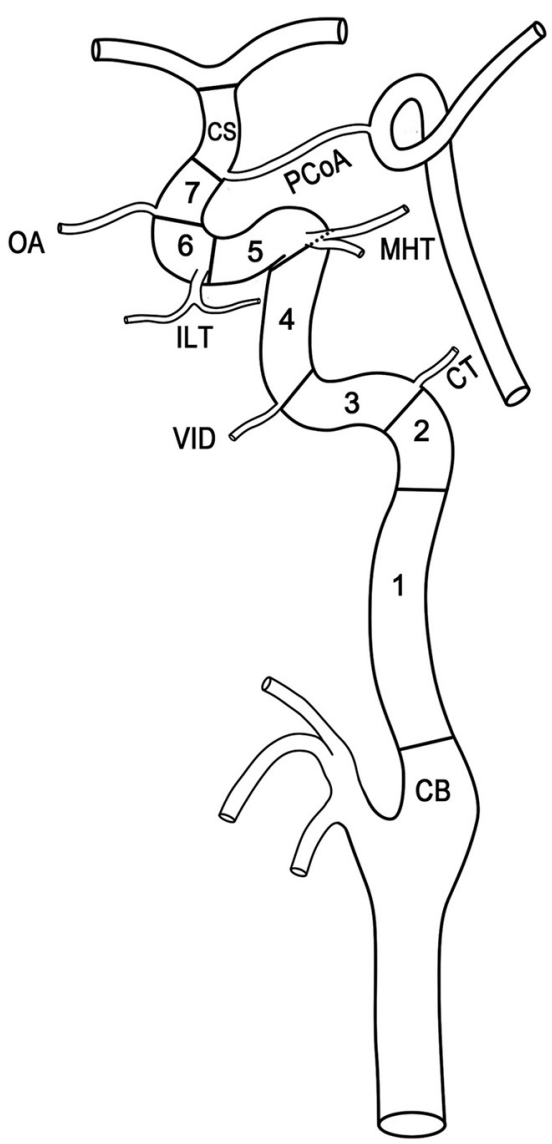

FIG 1. In addition to the 7 segments above, the carotid bulb to the PcomA origin, the segment of the ICA between the PcomA origin and ICA bifurcation before the MCA and anterior cerebral artery, is designated as the CS or distal ICA in adults. ILT indicates inferolateral trunk; MHT, meningohypophyseal trunk; VID, vidian artery; CT, caroticotympanic artery; CB, carotid bulb.

artery (OA), BA, and posterior cerebral artery (PCA) (P1 and P2 segments). The term "dysplasia" encompassed a variety of arterial abnormalities, including looping, coiling, ectasia, dolichoectasia, or simple dolichosis. In addition, the presence of lesions in the contralateral ICA, BA, and/or ipsi- and contralateral vertebral arteries was noted.

DSA, CTA, and MRA were used to analyze vessel stenoses and aneurysms. Stenosis was defined as any narrowing of the vessel lumen compared with the reference vessel as defined in the Warfarin-Aspirin Symptomatic Intracranial Disease trial method. ${ }^{10}$ The aneurysm was defined as an eccentric bulging of the vessel wall within the dolichoectatic segment, and the diagnosis was reached with the consensus of 2 doctors (D.H.L. and Z.Y.J.). CT was used to analyze vessel wall calcification. Contrast-enhanced MR imaging of the vessel wall was used to analyze vessel wall characteristics, including enhancement, wall thickening, and luminal narrowing. Brain perfusion SPECT was used to evaluate whether a stenosis or tortuous ICA led to a decrease in brain perfusion.

The patients were divided into 2 groups according to the PHACE syndrome diagnostic criteria: the group positive for PHACE, PHACE $(+)$, and the group negative for PHACE, PHACE $(-)$. The PHACE $(+)$ group included both confirmed and possible cases of PHACE syndrome. The Fisher exact test was used to assess differences between the groups. Descriptive analyses were 


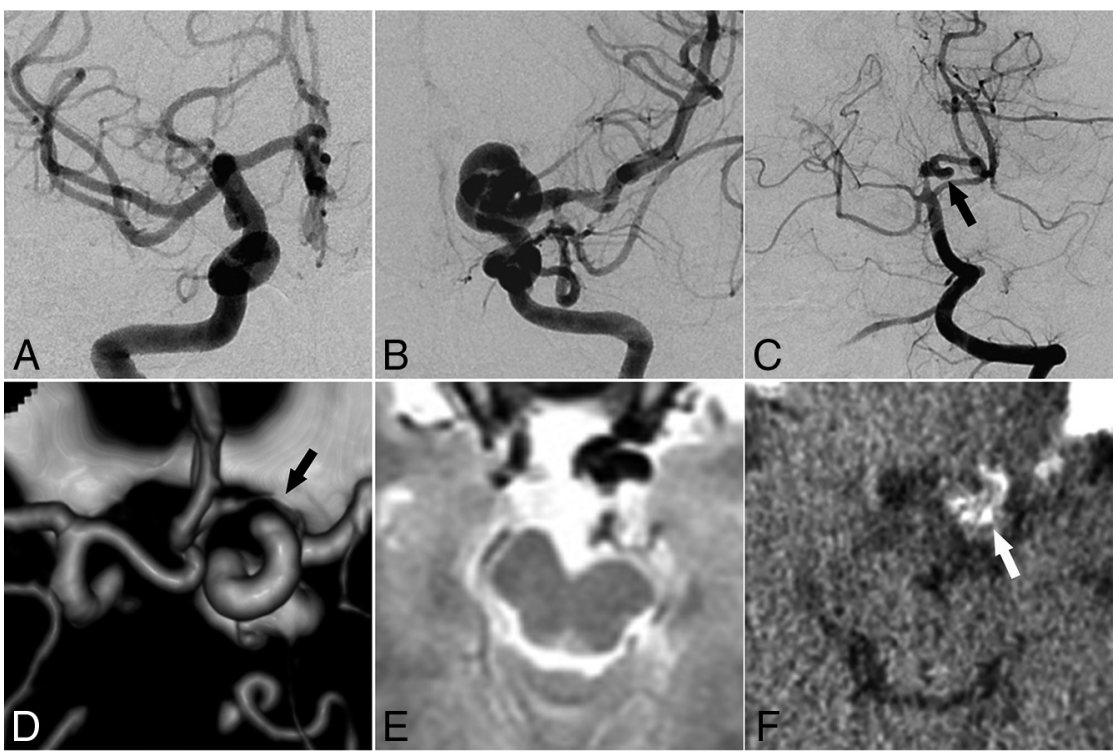

FIG 2. Representative images of ICDE in a patient without PHACE syndrome (patient 6, a 28year-old woman). $A$ and $B$, Bilateral ICA angiography shows a tangled arterial mass of the left distal ICA and no observable left $A l$ segment. $C$, Left vertebral artery angiography shows a dolichoectasia of the left P1 and P2 segments (arrow). D, CT angiography shows hypoplasia of the left A1 segment (arrow). E, T2-weighted image of the brain shows hypoplasia of the left midbrain. F, CT image of vessel wall calcification in the left distal ICA (arrow).

Table 1: Arterial components and brain structures surrounding the distal ICA on the affected side versus the contralateral side

\begin{tabular}{llcc}
\hline & Affected Side $(\boldsymbol{n}=\mathbf{2 0})$ & Contralateral Side $(\boldsymbol{n}=\mathbf{2 0})$ & $\boldsymbol{P V}$ Value $^{\mathbf{a}}$ \\
\hline A1 & Dysplasia $=15(75.0 \%)$ & Dysplasia $=1(5.0 \%)$ & .001 \\
M1 & Dysplasia $=2(10.0 \%)$ & Dysplasia $=0(0.0 \%)$ & $.487^{\mathrm{b}}$ \\
OA & Ectopic $=5(25.0 \%)$ & Ectopic $=1(5.0 \%)$ & .219 \\
AchoA & Dysplasia $=5(25.0 \%)$ & Dysplasia $=0(0.0 \%)$ & $.047^{\mathrm{b}}$ \\
PcomA & Dysplasia $=14(70.0 \%)$ & Dysplasia $=3(15.0 \%)$ & .003 \\
BA/PCA & Dysplasia $=14(70.0 \%)$ & Dysplasia $=1(5.0 \%)$ & $<.001$ \\
Midbrain & Hypoplasia $=9(45.0 \%)$ & Hypoplasia $=0(0.0 \%)$ & $.001^{\mathrm{b}}$ \\
\hline
\end{tabular}

Note:-Al indicates a segment of the anterior cerebral artery; M1, a segment of the MCA. BA/PCA indicates either BA or PCA, or both.

${ }^{a}$ McNemar test.

${ }^{\mathrm{b}}$ Fisher exact test.

performed to evaluate possible relationships among the following features: age, wall calcification, vessel wall enhancement, stenosis, and aneurysm.

\section{RESULTS}

The basic demographic information, presenting symptoms, involved arteries around the distal ICA, acquired changes in affected segments, and PHACE diagnostic statuses of all patients are summarized in the On-line Table. The patients included 18 females and 2 males with a median age of 43.5 years (range, 7-73 years) with varying clinical symptoms that did not appear to be directly related to the arterial abnormality. A systemic review revealed that 3 patients had hypertension, whereas none had systemic vasculitis or autoimmune disease.

All patients had the unique feature, segmental elongation and tortuosity of the distal ICA, resulting in a tangled arterial mass (Fig 2). No differences were observed between the sides of onset (right, 11/20). Two patients presented with bilateral distal ICA dolichoectasia. In these 2 patients, we defined the side with more severe dolichoectasia as the ipsilateral side, while the other side was the contralateral side. The involved ICA segments ranged from segments 2 to 7 , with a mean \pm SD of $3.5 \pm 1.6$ segments. Fourteen patients had BA $(n=1)$ or ipsilateral PCA $(n=13)$ involvement [BA/ PCA $(+)$ ]. We observed a significant correlation between ipsilateral A1, PcomA, BA/PCA, and midbrain hypoplasia and segmental ICDE of the distal ICA, unlike the contralateral side (Table 1).

In 9 of the 20 patients, the ipsilateral midbrain was smaller than the contralateral midbrain (Fig 2E). Four and 7 patients were found to have confirmed or possible PHACE syndrome, respectively, when midbrain hypoplasia was excluded as a major or minor criterion for a PHACE syndrome diagnosis (posterior fossa anomaly). The inclusion of this criterion resulted in 4 and 11 patients with confirmed and possible PHACE syndrome, respectively. No significant differences in arterial component dysplasia (including A1, anterior communicating artery, M1, AchoA, PcomA, OA, BA, and PCA) and brain structures were observed between patients positive and negative for PHACE (Table 2).

Calcification was detected in 16 patients with a mean age of 45.9 years; the remaining 4 patients without calcification had a mean age of 17.3 years. Significantly progressive calcification was observed in 1 patient with progressive arterial stenosis during an interval of 10 years (patient 9; age range, 42-52 years). Stenosis was detected in 11 patients with a mean age of 42.1 years; the 9 patients without stenosis had a mean age of 37.9 years. None of the patients had an ischemic stroke event, and none of the 12 patients who underwent brain perfusion SPECT had hypoperfusion. Aneurysms were detected in 15 patients with a mean age of 38.1 years; the 5 patients without aneurysms had a mean age of 48.8 years.

Ten patients underwent contrast-enhanced high-resolution MR imaging of the vessel wall, which detected enhancement in 6 patients with a mean age of $22.8 \pm 13.2$ years; the remaining 4 patients had a mean age of $57.0 \pm 9.2$ years $(P<.001)$. One patient (patient 1, Fig 3) exhibited vessel wall enhancement at both 8 and 12 years of age, with no major change in the enhancement pattern. Vessel wall imaging revealed that the stenotic segment exhibited either an eccentric or concentric wall thickening (Fig 4) as well as a potential enhancement of the parent artery and aneurysm neck (Fig 5).

AJNR Am J Neuroradiol 39:817-23 May 2018 www.ajnr.org 819 
Table 2: Arterial components around the distal ICA on the affected side in patients with and without PHACE syndrome ${ }^{a}$

\begin{tabular}{lllc}
\hline & PHACE $(+)(\boldsymbol{n}=11)$ & PHACE $(-)(\boldsymbol{n}=9)$ & P Value $^{\text {b }}$ \\
\hline A1 & Dysplasia $=8(72.7 \%)$ & Dysplasia $=7(77.8 \%)$ & 1.000 \\
M1 & Dysplasia $=0(0.0 \%)$ & Dysplasia $=2(22.2 \%)$ & .189 \\
OA & Ectopic $=1(9.1 \%)$ & Ectopic $=4(44.4 \%)$ & .127 \\
AchoA & Dysplasia $=3(27.3 \%)$ & Dysplasia $=2(22.2 \%)$ & 1.000 \\
PcomA & Dysplasia $=7(63.6 \%)$ & Dysplasia $=7(77.8 \%)$ & .642 \\
Midbrain & Dysplasia $=5(45.5 \%)$ & Dysplasia $=4(44.4 \%)$ & 1.000 \\
\hline
\end{tabular}

${ }^{a}$ PHACE includes cases of confirmed and possible PHACE syndrome; a small midbrain was not used as a criterion.

${ }^{\mathrm{b}}$ Fisher exact test.
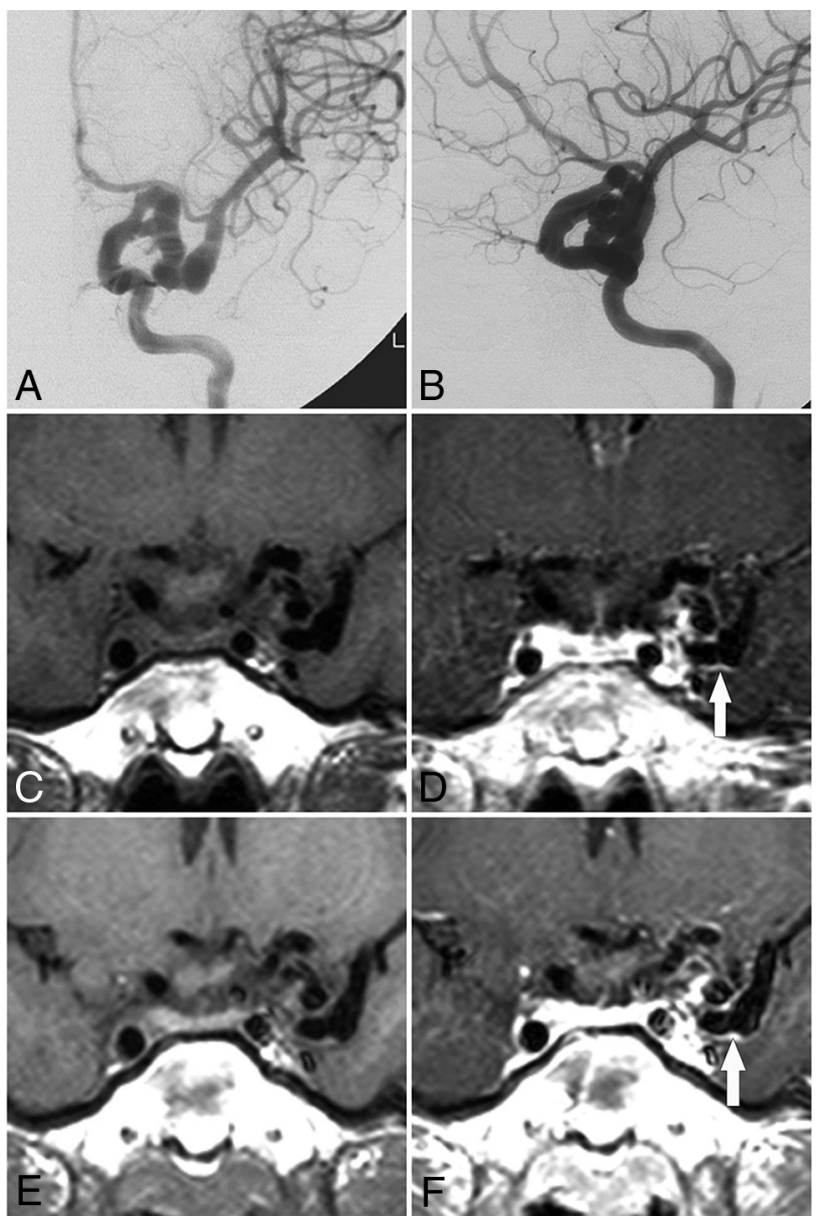

FIG 3. Evolution of vessel wall enhancement with aging in a patient with PHACE syndrome (patient 1, an 8-year-old girl). $A$ and $B$, Left ICA angiography (anteroposterior and oblique views) reveals tortuosity in the left distal ICA with a small and long Al segment. $C$ and $D$, Noncontrast and contrast-enhanced vessel wall imaging at 8 years of age demonstrates vessel wall enhancement and slight vessel wall thickening (arrow in $D$ ). $E$ and $F$, Vessel wall imaging at 12 years of age shows a similar vessel wall enhancement pattern (arrow in F).

\section{DISCUSSION}

In our series of patients across a wide range of ages, we observed striking elongation and tortuosity of the distal ICA, regardless of the PHACE diagnostic status. We further observed that compared with the contralateral side, the ipsilateral arterial components and brain structures around the affected distal ICA more frequently showed dysplasia. Furthermore, the affected vessels had various manifestations, including stenosis, aneurysm, calcification, and vessel wall enhancement, and the disease evolved slowly with age according to follow-up radiologic imaging.

\section{Embryologic Pathogenesis of ICDE of the Distal ICA}

During the embryologic period of cerebral artery formation, angiogenesis is mainly driven by hypoxia and related growth factors in the target tissue. ${ }^{11,12}$ We hypothesized that exposure of a specific segment of the intracranial artery to a vasculogenetic trigger resulted in arterial lengthening. According to the ICA developmental anatomy proposed by Lasjaunias and Santoyo-Vazquez, ${ }^{13}$ the ICA branches into a cranial and caudal rami at stages I and II (3.5-4 weeks); the former gives rise to the anterior cerebral artery and AchoA, whereas the latter gives rise to the PcomA, P1 segment, and upper BA. In this study, dolichoectasia at the anterior (A1 segment, AchoA, and CS segment) and posterior (PcomA, P1 segment, and upper BA) divisions of the ICA suggested that these regions were triggered during embryonic development. We observed no involvement of the superior cerebellar artery in our cases (data not shown); because the superior cerebellar artery existed before ICA branching, the vasculogenetic event may have occurred after the appearance of superior cerebellar artery. ${ }^{13}$ Although the remaining PCA (segments P2-P4) comprises the posterior choroidal branch of the caudal ramus at stage $\mathrm{V}$, the involvement of the $\mathrm{P} 2$ segment in several cases suggests that the PCA precursor had also been affected. ${ }^{13}$

\section{PHACE Syndrome}

Cerebral arterial anomalies are observed in $91 \%$ of patients with PHACE syndrome, ${ }^{14}$ and previous studies of PHACE syndrome have reported a presentation of dolichoectasia of the internal carotid arteries similar to that observed in our cases. ${ }^{2,4,15,16}$ Therefore, we searched for common features between our cases and PHACE syndrome cases.

First, we observed a strong female predominance in our patient group in agreement with previous studies of PHACE syndrome (up to 8:1). ${ }^{15,17,18}$ Second, the timing of PHACE syndrome was consistent with our speculated time course. Several studies of PHACE syndrome have reported that the teratogenic influence might occur from gestational weeks 3 to $5.5,{ }^{8,14}$ concurrent with the regression of the embryonic capillary bed and active stemming of the craniocervical vasculature. Therefore, any influences on these 2 processes may result in a cutaneous hemangioma and trigeminal artery persistence. ${ }^{19}$

Third, several vascular anomalies have been reported in both patients with PHACE syndrome and in our patient group. In a previous study, A1 hypoplasia was reported as an intracranial anomaly affecting 8 of 12 patients with PHACE syndrome; this is similar to the findings of our study $(15 / 20) .{ }^{20}$ ICDE of the ICA was accompanied by dolichoectasia of the posterior circulation in 6 of 7 patients with PHACE syndrome in a previous study. ${ }^{20}$ An aberrant origin or course of the principal cerebral arteries, a major or minor PHACE syndrome criterion, was observed in 9 of 20 patients (45\%; 5 ectopic ophthalmic arteries and 4 other arteries).

Fourth, 4 of the 20 patients in this study met the diagnostic criteria of PHACE syndrome, and an additional 7 patients were classified as possible cases of PHACE syndrome. In addition, some 

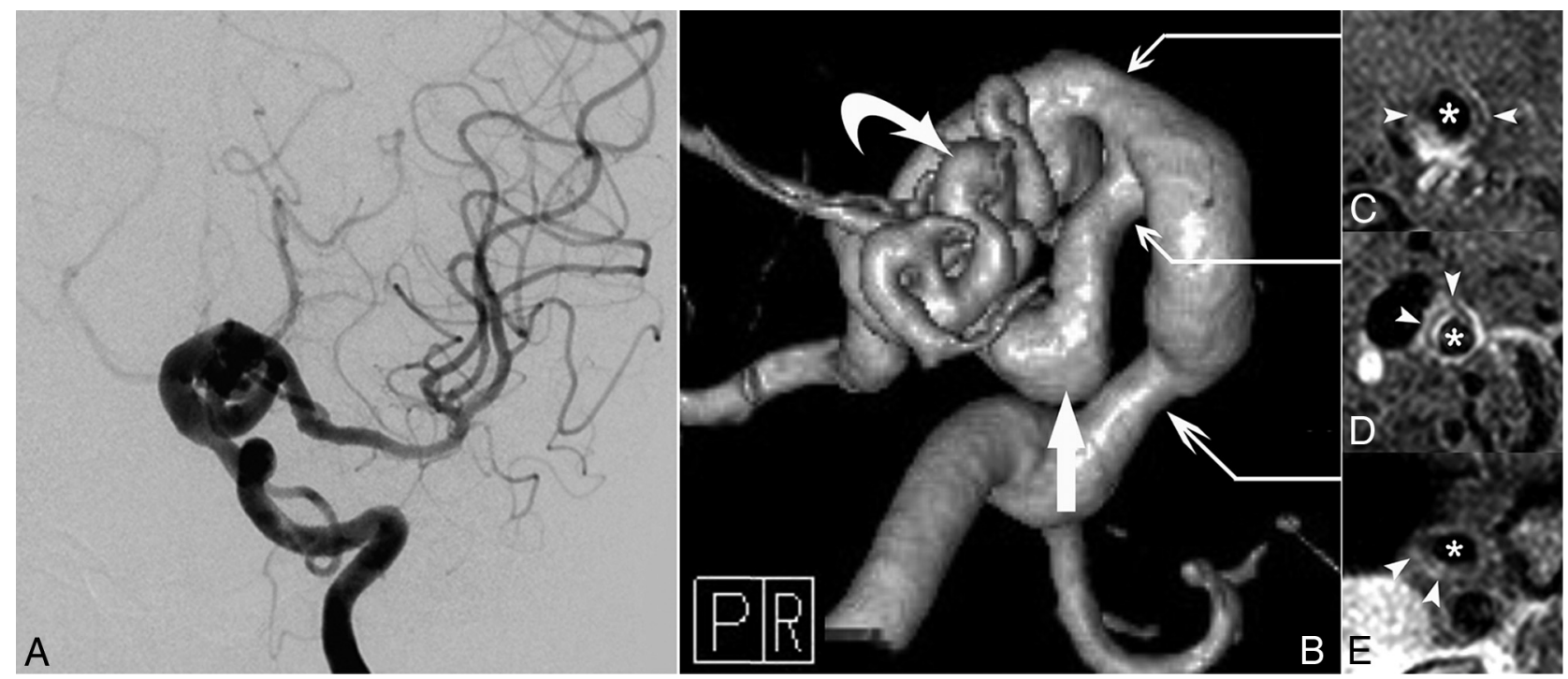

FIG 4. Vessel wall enhancement at the stenotic segment in a patient with possible PHACE syndrome (patient 5, a 23-year-old woman). $A$ and $B$, Left ICA angiography and TOF-MRA reveal dolichoectasia of the left distal ICA, left PcomA (arrow), and left AchoA (curved arrow). C-E, Section images reveal the vessel wall thickening and enhancing patterns of each corresponding stenotic segment (arrowheads indicate the vessel wall thickening and asterisks indicate vessel lumen; $C$, sagittal plane; $D$ and $E$, axial plane). $P$ indicates posterior; $R$, right.
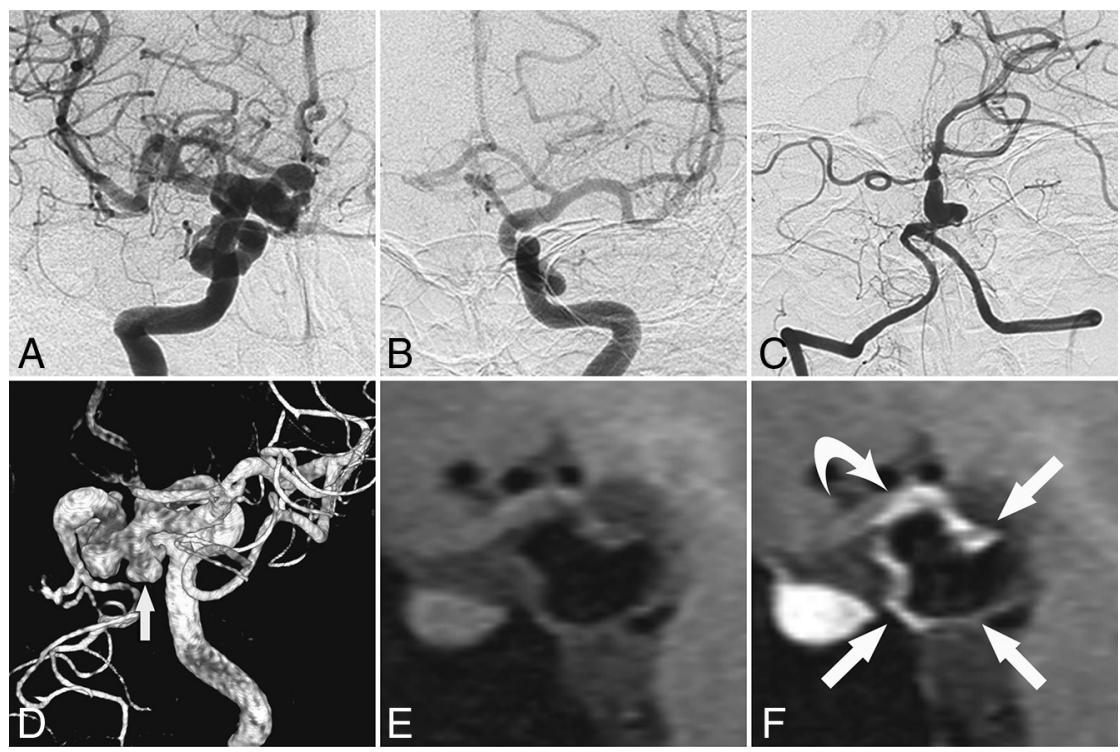

FIG 5. Vessel wall enhancement at the aneurysm wall and the parent artery wall in a patient without PHACE syndrome (patient 11, a 45-year-old woman). A-C, Cerebral angiography shows dolichoectasia in the right distal ICA and basilar artery. D, 3D-DSA shows dolichoectasia in the right distal ICA and right PcomA as well as the formation of multiple aneurysms in the right PcomA (arrow). E and F, Non-contrast-enhanced and contrast-enhanced vessel wall imaging shows enhancement of the aneurysm wall (arrows in F) and the parent artery wall (curved arrow in $F$ ).

patients exhibited ipsilateral midbrain hypoplasia. Because a lack of direct contact with the adjacent vessel does not support compression-induced midbrain deformation (Fig 2E), we suspected that dolichoectasia of the arteries feeding the midbrain (BA, P1, or P2) mildly altered the blood supply and caused further hypoplasia. In 1 patient with PHACE (patient 10), hypoplasia of the ipsilateral cerebellum and the ipsilateral midbrain was found to coexist, suggesting that these 2 structural anomalies shared a common origin. Therefore, if a smaller midbrain was defined as a posterior fossa anomaly associated with PHACE syndrome, an additional 4 patients in our study would meet the criteria for possible PHACE syndrome. Such lesions might broaden the PHACE syndrome phenotype. According to a study by Heyer et al, ${ }^{20}$ moderate effacement of the right pons (Fig $2 \mathrm{C}$ in the article by Heyer et al) and cerebral peduncle along with corresponding vascular anomalies was observed via MR imaging in a patient with PHACE syndrome. However, the author did not propose this finding as an anomaly. ${ }^{20}$

In our study, we found no significant differences in arterial component dysplasia and brain structures between patients positive and negative for PHACE (Table 2), suggesting that the 2 groups of patients share the same features and pathogenesis. Furthermore, although we did not detect an obvious cutaneous hemangioma in many of our patients, a previous study found that very small cutaneous hemangiomas might be absent or regress spontaneously without prior recognition or reporting. ${ }^{4}$ The abovementioned aspects raise the intriguing possibility that a marked ICDE of the ICA might indicate an otherwise-unrecognized partial phenotypic expression of PHACE syndrome. However, the spontaneous regression of cutaneous hemangiomas at an early age may cause the underestimation of the incidence of PHACE syndrome in this group of patients, which further induces underestimation of the relationship between the ICDE and PHACE syndrome.

\section{Acquired Changes in the Involved Arterial Wall}

Normally, an abnormal mural angiogenesis likely causes an increase in the luminal caliber because the correct remodeling signals induce apoptosis of the unnecessary vessel wall components. 
A lack of remodeling causes centripetal and longitudinal proliferation and luminal reduction; however, this might also cause ectasias, elongated arteries, and aneurysms. ${ }^{13}$ Although many cases involving ICDE of the ICA, with or without PHACE syndrome, have been reported, little is known about the evolution of vessel wall lesions and relevant complications. Results from a follow-up study of vessel lesions may provide valuable prognostic information.

Progressive cerebral arterial stenosis and arterial occlusion and a Moyamoya-like vasculopathy leading to stroke have been described in infants with PHACE syndrome. ${ }^{2,20}$ This progressive cerebral vasculopathy corresponds with the proliferative phase of hemangioma growth, and as a result, the average age of experiencing stroke among patients with PHACE syndrome is 8.8 months. ${ }^{21}$ However, no ischemic stroke events were reported in our present study, even among patients with a Moyamoya-like vasculopathy (patients $4,8,12$ ). Notably, we observed vessel wall enhancement more frequently among young patients than older patients, indicating a regression in inflammation with aging. Accordingly, we speculate that most arterial stenoses and occlusions formed within a short time during the prenatal or infant stage. Patients with mild lesions might pass through that period asymptomatically, and adult PHACE syndrome diagnoses may be incidental. ${ }^{1,22}$ Due to the long-standing nature of the lesion, there is a good chance of good collateral circulation formation secondary to arterial stenoses, which may present as nonsymptomatic stenoocclusive disease.

We further propose that ICDE of the distal ICA, with or without PHACE syndrome, might stabilize after a period of rapid progression. Bracken et $\mathrm{al}^{15}$ followed up several cases of PHACE in neurodevelopmentally healthy patients for 1-12 years. McLaughlin et $\mathrm{al}^{5}$ reported a 24-year-old female patient with a pure arterial malformation involving the distal ICA, PcomA, and PCA that was found on a CT scan obtained to determine the cause of a headache. When this patient was later followed up at 54 years of age, the abnormal vessels had not changed with time on MR images, and no symptoms relevant to the abnormal vessels were reported during the 31-year interval. ${ }^{5}$ Similarly, our review of imaging data collected during a long follow-up demonstrated a slow evolution of vessel wall enhancement (patient 1) and slow progression of both calcification and stenosis (patient 9). The vessel wall enhancement in patient 1 could be explained by the immature nature of the affected vessel wall, which may increase the permeability of the endothelium, with contrast leakage from the lumen into the arterial wall, and may be simultaneously associated with an atherosclerotic-like process in the dysplastic segments.

\section{Treatment and Follow-Up}

Many cases of ICDE with tortuous ICAs were identified incidentally, without relevant symptoms ${ }^{5,7,15}$; in these cases, the lesions appeared stable on follow-up images and the patients did not receive medical treatment. However, several reports and our observations suggested the need for regular imaging follow-up as well as medication in some cases. However, no specific treatment exists for dolichoectasia, and the surgical and medical therapies used to treat this condition have not been systematically evalu- ated. Although anticoagulation and antiplatelet therapies might help in preventing an ischemic episode, some studies have indicated that aspirin and warfarin or both do not effectively reduce the stroke recurrence rates in patients with dolichoectasia and might increase the risk of hemorrhage in this population. ${ }^{23}$ However, we note that these previous data were all with respect to the BAs.

Treatment for PHACE syndrome should address the aforementioned symptoms. ${ }^{2}$ Corticosteroids and interferon have been previously used to treat hemangiomas associated with PHACE syndrome; however, their efficacy in the treatment of acute-phase vessel wall inflammation remains unknown. ${ }^{16}$ Occasionally, a pial synangiosis procedure has been suggested for severe stenosis or occlusion of the distal ICA. ${ }^{20}$

We believe that attention should be paid to several cases in this study. One patient (patient 1) exhibited simultaneous vessel wall enhancement and calcification at 7 years of age, leading to our hypothesis that the affected vessel wall was prone to atherosclerosis formation and secondary calcification. Questions also remain regarding the use of antiatherosclerosis therapies in young patients. Another patient (patient 11, Fig 5) exhibited vessel wall enhancement in an aneurysm and its parent artery, which may be a risk factor for aneurysm rupture; accordingly, a pre-emptive aneurysm embolization was performed. ${ }^{24}$ In another patient (patient 16), asymptomatic ICDE of the right ICA and hypoplasia of the right A1 segment were detected at 54 years of age, and a blood flow-related aneurysm of the left anterior communicating artery was observed at 69 years of age. This patient was later treated with coiling embolization. Therefore, we suggested a follow-up comprising regular angiography studies (CTA or MRA) to demonstrate overall luminal changes and, if possible, vessel wall imaging to detect inflammation in the lesion.

\section{Limitations}

This study had several limitations. First, we found it difficult to objectively define "segmental dolichoectasia." To overcome this problem, we included only cases with noncontroversial elongation and unusual tortuosity relative to other segments or the contralateral ICA. Accordingly, we might have skipped many mild elongation cases and underestimated the number of relevant cases. Second, the definition of segmental arterial tortuosity is rather subjective. Lasjaunias et $\mathrm{al}^{9}$ defined the ICA segments according to embryogenic evolution. In this study, we considered ICDE of the distal ICA to be a congenital disease that may occur segmentally. Although we used this system to describe the observed lesions, we were unable to conclude that the lesions could be attributed to a similar congenital origin. Third, 2 patients in our study had bilateral ICA involvement, which has also been reported in patients with PHACE syndrome. However, a satisfactory interpretation of the bilateral pathogenesis could not be attained. ${ }^{20}$

\section{CONCLUSIONS}

The segmental nature of the striking elongation and tortuosity of the distal ICA suggests a type of congenital lesion representing either a sporadic phenomenon or an arterial change associated with PHACE syndrome. Similar arterial changes were observed in 
vascular segments adjacent to the lesions, particularly in the ipsilateral proximal PCA. Imaging findings of affected patients demonstrated various mural abnormalities with a benign clinical course.

\section{REFERENCES}

1. Burch EA, Garzon MC, Parikh A, et al. A 65-year-old woman diagnosed with PHACE syndrome. Pediatr Dermatol 2013;30:e153-56 CrossRef Medline

2. Baccin CE, Krings T, Alvarez H, et al. A report of two cases with dolichosegmental intracranial arteries as a new feature of PHACES syndrome. Childs Nerv Syst 2007;23:559-67 CrossRef Medline

3. Metry DW, Dowd CF, Barkovich AJ, et al. The many faces of PHACE syndrome. J Pediatr 2001;139:117-23 CrossRef Medline

4. Rossi A, Bava GL, Biancheri R, et al. Posterior fossa and arterial abnormalities in patients with facial capillary haemangioma: presumed incomplete phenotypic expression of PHACES syndrome. Neuroradiology 2001;43:934-40 CrossRef Medline

5. McLaughlin N, Raychev R, Duckwiler G, et al. Pure arterial malformation of the posterior cerebral artery: importance of its recognition. J Neurosurg 2013;119:655-60 CrossRef Medline

6. Yuh SJ, Alkherayf F, Lesiuk H. Dolichoectasia of the vertebral basilar and internal carotid arteries: a case report and literature review. Surg Neurol Int 2013;4:153 CrossRef Medline

7. Nakahara I, Taki W, Tanaka M, et al. Dolichoectasia of the middle cerebral artery: case report. Neurol Med Chir (Tokyo) 1995;35: 822-24 CrossRef Medline

8. Metry D, Heyer G, Hess C, et al; PHACE Syndrome Research Conference. Consensus statement on diagnostic criteria for PHACE syndrome. Pediatrics 2009;124:1447-56 CrossRef Medline

9. Lasjaunias PL, Berenstein A, Ter Brugge KG. Surgical Neuroangiography: Clinical Vascular Anatomy and Variations. 2nd ed. Vol. 1. Berlin: Springer-Verlag; 2001

10. Samuels OB, Joseph GJ, Lynn MJ, et al. A standardized method for measuring intracranial arterial stenosis. AJNR Am J Neuroradiol 2000;21:643-46 Medline

11. Menshawi K, Mohr JP, Gutierrez J. A functional perspective on the embryology and anatomy of the cerebral blood supply. J Stroke 2015;17:144-58 CrossRef Medline
12. Plate KH. Mechanisms of angiogenesis in the brain. J Neuropathol Exp Neurol 1999;58:313-20 CrossRef Medline

13. Lasjaunias P, Santoyo-Vazquez A. Segmental agenesis of the internal carotid artery: angiographic aspects with embryological discussion. Anat Clin 1984;6:133-41 CrossRef Medline

14. Haggstrom AN, Garzon MC, Baselga E, et al. Risk for PHACE syndrome in infants with large facial hemangiomas. Pediatrics 2010; 126:e418-26 CrossRef Medline

15. Bracken J, Robinson I, Snow A, et al. PHACE syndrome: MRI of intracerebral vascular anomalies and clinical findings in a series of 12 patients. Pediatr Radiol 2011;41:1129-38 CrossRef Medline

16. Vermeer $S$, van Oostrom CG, Boetes $C$, et al. A unique case of PHACES syndrome confirming the assumption that PHACES syndrome and the sternal malformation-vascular dysplasia association are part of the same spectrum of malformations. Clin Dysmorphol 2005;14:203-06 CrossRef Medline

17. Metry DW, Haggstrom AN, Drolet BA, et al. A prospective study of PHACE syndrome in infantile hemangiomas: demographic features, clinical findings, and complications. Am J Med Genet A 2006; 140:975-86 Medline

18. Oza VS, Wang E, Berenstein A, et al. PHACES association: a neuroradiologic review of 17 patients. AJNR Am J Neuroradiol 2008;29: 807-13 CrossRef Medline

19. Pascual-Castroviejo I, Viaño J, Moreno F, et al. Hemangiomas of the head, neck, and chest with associated vascular and brain anomalies: a complex neurocutaneous syndrome. AJNR Am J Neuroradiol 1996; 17:461-71 Medline

20. Heyer GL, Dowling MM, Licht DJ, et al. The cerebral vasculopathy of PHACES syndrome. Stroke 2008;39:308-16 CrossRef Medline

21. Heyer GL, Millar WS, Ghatan S, et al. The neurologic aspects of PHACE: case report and review of the literature. Pediatr Neurol 2006;35:419-24 CrossRef Medline

22. Arora SS, Plato BM, Sattenberg RJ, et al. Adult presentation of PHACES syndrome. Interv Neuroradiol 2011;17:137-46 CrossRef Medline

23. Passero SG, Calchetti B, Bartalini S. Intracranial bleeding in patients with vertebrobasilar dolichoectasia. Stroke 2005;36:1421-25 CrossRef Medline

24. Edjlali M, Gentric JC, Regent-Rodriguez C, et al. Does aneurysmal wall enhancement on vessel wall MRI help to distinguish stable from unstable intracranial aneurysms? Stroke 2014;45:3704-06 CrossRef Medline 